\title{
Sur les mauvais facteurs locaux des fonctions $L$ attachées aux surfaces abéliennes et surfaces K-3
}

\author{
par \\ Jean Claude Douai (Lille)
}

Soient $k$ un corps de nombres, $X$ une variété projective, lisse, définie sur $k, l$ un premier $\neq 2$. L'un des objectifs de ce travail est d'étudier les facteurs locaux

$$
L_{v}(X, s)=\operatorname{det}\left(1-\operatorname{Fr}_{v}(N v)^{-s} \mid H^{2}\left(\bar{X}, Q_{l}\right)^{I_{v}}\right)^{-1}
$$

de la fonction $L$ attachée à $X$ en les places $v$ où il y a mauvaise réduction $\left(\mathrm{Fr}_{v}=\right.$ Frobenius géométrique en $v, I_{v}=$ inertie en $\left.v\right)$. En particulier, nous déterminons au $\S I I I$ l'ordre en $s=0$ du pôle de $L_{v}(X, s)$ quand $X$ est une surface K-3 ou encore une surface abélienne d'un certain type. L'ordre de ce pôle dépend étroitement du type de dégénérescence en $v$ de la variété $X$ et est liée au calcul du $H_{\mathrm{et}}^{4}\left(X / k_{v}, Q_{l} / Z_{l}(3)\right)$ qui avait été entamé dans [2]. Les ingrédients essentiels sont les résultats galoisiens de Jannsen [3] et les résultats de Kulikov-Shafarevich sur les dégénérescences des surfaces [5]. Dans le paragraphe IV, nous globalisons les résultats précédents en calculant le $H_{\text {et }}^{4}\left(X / k, Q_{l} / Z_{l}(3)\right)$ pour une surface $\mathrm{K}-3 X$.

Je tiens à remercier le referee pour ses corrections et ses observations.

Dans toute la suite, la topologie considérée sera la topologie étale.

Notations. Sauf dans le dernier paragraphe IV où $k$ sera global, $k=$ $k_{v}$ désignera un corps $p$-adique, $v$ la valuation qui lui est attachée, $G=$ $G_{k}=G_{k_{v}}$ son groupe de Galois, $I=I_{v} \subset G_{k_{v}}$ son groupe d'inertie, $\operatorname{Fr}_{v}$ son Frobenius géométrique, $k_{0}$ son corps résiduel. $X$ désignera une variété projective, lisse, définie sur $k$ et $l$ un premier différent de $p$. On écrit $\bar{X}=$ $X \otimes_{k} \bar{k}$.

I. Nous avons la suite spectrale de descente de Hochschild-Serre :

$$
E_{2}^{p, q}=H^{p}\left(G_{k}, H^{q}\left(\bar{X}, Q_{l} / Z_{l}(n)\right)\right) \Rightarrow H^{p+q}\left(X, Q_{l} / Z_{l}(n)\right),
$$

qui donne, pour $r \geq 2$ et $n$ entier quelconque, la suite exacte 


$$
\begin{aligned}
& \text { (1) } \quad H^{r-1}\left(\bar{X}, Q_{l} / Z_{l}(n)\right)^{G} \rightarrow H^{2}\left(k, H^{r-2}\left(\bar{X}, Q_{l} / Z_{l}(n)\right)\right) \\
& \rightarrow H^{r}\left(X, Q_{l} / Z_{l}(n)\right)_{0} \rightarrow H^{1}\left(k, H^{r-1}\left(\bar{X}, Q_{l} / Z_{l}(n)\right)\right) \rightarrow 0 \\
& \text { où } H^{r}\left(X, Q_{l} / Z_{l}(n)\right)_{0}=\operatorname{Ker}\left\{H^{r}\left(X, Q_{l} / Z_{l}(n)\right) \rightarrow H^{r}\left(\bar{X}, Q_{l} / Z_{l}(n)\right)^{G}\right\} .
\end{aligned}
$$

Par la conjecture de Jannsen ([3], p. 342), $H^{2}\left(k, H^{r-2}\left(\bar{X}, Q_{l} / Z_{l}(n)\right)\right)$ est fini si (a) $r-2+1<n$, (b) $r-2+1>2 n$.

Par exemple, si $r=4$, ceci donne (a) $3<n$, (b) $3>2 n$, auquel cas $H^{2}\left(k, H^{2}\left(\bar{X}, Q_{l} / Z_{l}(n)\right)\right)$ est fini pour $n \neq 2$ ou 3 .

Il existe une formulation équivalente de la conjecture de Jannsen (cf. loc. cit.) : on pose $m=i+1-n$ avec $i=r-2$, alors $H^{i}\left(\bar{X}, Q_{l}(m)\right)^{G} \neq 0$ au plus pour $0 \leq m \leq(i+1) / 2$.

Dans le cas de notre exemple $r=4$, ceci donne: $H^{r-2}\left(\bar{X}, Q_{l}(m)\right)^{G_{k}} \neq 0$ au plus seulement pour $m=0$ ou 1 . Pour $r=4$, nous aurons donc seulement à considérer :

(i) $n=3$ ou $m=0$,

(ii) $n=2$ ou $m=1$.

Par le lemme 11(b) de Jannsen [3],

$$
\begin{aligned}
& \operatorname{dim} H^{2}\left(k, H^{2}\left(\bar{X}, Q_{l} / Z_{l}(n)\right)\right)=\operatorname{dim}_{Q_{l}} H^{2}\left(\bar{X}, Q_{l}(n-1)\right)_{G} \\
& \quad=\operatorname{dim}_{Q_{l}} H^{2}\left(\bar{X}, Q_{l}(m)\right)^{G} \leq-\operatorname{ord}_{s=m} L_{v}\left(H^{2}\left(X, Q_{l}\right), s\right)
\end{aligned}
$$

avec égalité si le Frobenius géométrique $\mathrm{Fr}_{v}$ agit semi-simplement sur $H^{2}\left(\bar{X}, Q_{l}\right)^{I_{v}}$. Le fait que $\operatorname{Fr}_{v}$ agit semi-simplement sur $H^{\nu}\left(\bar{X}, Q_{l}\right)^{I_{v}}, \nu \geq 1$, est précisément une conjecture de Serre et Grothendieck. $L_{v}\left(H^{2}\left(\bar{X}, Q_{l}\right), s\right)$ n'admet donc de pôles qu'en $s=0$ ou 1 si l'on admet d'une part la conjecture de Jannsen, d'autre part celle de Serre-Grothendieck.

La suite exacte (1) appliquée au cas $r=4$ donne les suites exactes suivantes (cf. $\mathrm{n}^{\circ} 3^{\prime}, \S I I$ de [2] où $X$ est supposé simplement connexe) :

(i) pour $n=3$ :

$$
\begin{aligned}
H^{3}\left(\bar{X}, Q_{l} / Z_{l}(3)\right)^{G} & \rightarrow H^{2}\left(k, H^{2}\left(\bar{X}, Q_{l} / Z_{l}(3)\right)\right) \\
& \rightarrow H^{4}\left(X, Q_{l} / Z_{l}(3)\right)_{0} \rightarrow H^{1}\left(k, H^{3}\left(\bar{X}, Q_{l} / Z_{l}(3)\right)\right) \rightarrow 0 .
\end{aligned}
$$

(Remarquer que $H^{4}\left(\bar{X}, Q_{l} / Z_{l}(3)\right) \simeq Q_{l} / Z_{l}(1)$, d'où l'on déduit que $H^{4}\left(\bar{X}, Q_{l} / Z_{l}(3)\right)^{G}$ est fini; $H^{4}\left(X, Q_{l} / Z_{l}(3)\right)$ et $H^{4}\left(X, Q_{l} / Z_{l}(3)\right)_{0}$ ont donc mêmes corangs);

(ii) pour $n=2$ :

$$
\begin{aligned}
H^{3}\left(\bar{X}, Q_{l} / Z_{l}(2)\right)^{G} & \rightarrow H^{2}\left(k, H^{2}\left(\bar{X}, Q_{l} / Z_{l}(2)\right)\right) \\
\rightarrow H^{4}\left(X, Q_{l} / Z_{l}(2)\right)_{0} & \rightarrow H^{1}\left(k, H^{3}\left(\bar{X}, Q_{l} / Z_{l}(2)\right)\right) \rightarrow 0 .
\end{aligned}
$$


II. Nous nous intéresserons surtout au cas $n=3$.

Proposition 1. Soit $X$ une surface projective, lisse, définie sur un corps p-adique $k=k_{v}$. Alors

$\operatorname{dim} H^{4}\left(X, Q_{l} / Z_{l}(3)\right)=\operatorname{dim}_{Q_{l}} H^{4}\left(X, Q_{l}(3)\right)=\operatorname{dim}_{Q_{l}} H^{2}\left(X, Q_{l}\right)$

$\geq \operatorname{dim}_{Q_{l}} H^{2}\left(\bar{X}, Q_{l}(2)\right)_{G}=\operatorname{dim}_{Q_{l}} H^{2}\left(\bar{X}, Q_{l}\right)^{G}=-\operatorname{ord}_{s=0} L_{v}\left(H^{2}\left(\bar{X}, Q_{l}\right), s\right)$,

cette dernière égalité étant valable si le Frobenius géométrique $\mathrm{Fr}_{v}$ agit semisimplement sur $H^{2}\left(\bar{X}, Q_{l}\right)^{I_{v}}$ ou si l'on admet la conjecture de SerreGrothendieck [resp. $\operatorname{dim} H^{4}\left(X, Q_{l} / Z_{l}(2)\right)_{0} \geq \operatorname{dim}_{Q_{l}} H^{2}\left(\bar{X}, Q_{l}(1)\right)^{G}=$ $-\operatorname{ord}_{s=1} L_{v}\left(H^{2}\left(\bar{X}, Q_{l}\right), s\right)$ si $\left.\ldots\right]$.

Démonstration. L'égalité $\operatorname{dim}_{Q_{l}} H^{4}\left(X, Q_{l}(3)\right)=\operatorname{dim}_{Q_{l}} H^{2}\left(X, Q_{l}\right)$ résulte de la dualité de Poincaré-Tate entre $H^{i}\left(X, Q_{l}\right)$ et $H^{6-i}\left(X, Q_{l}(3)\right)$ (cf. [2], §II, no $\left.2^{\prime}\right)$. D'où $\operatorname{dim} H^{4}\left(X, Q_{l} / Z_{l}(3)\right)=\operatorname{dim}_{Q_{l}} H^{4}\left(X, Q_{l}(3)\right)=$ $\operatorname{dim}_{Q_{l}} H^{2}\left(X, Q_{l}\right)$. L'égalité $\operatorname{dim}_{Q_{l}} H^{2}\left(\bar{X}, Q_{l}(2)\right)_{G}=\operatorname{dim}_{Q_{l}} H^{2}\left(\bar{X}, Q_{l}\right)^{G}$ provient de la dualité de Poincaré :

$$
H^{2}\left(\bar{X}, Q_{l}(2)\right) \times H^{2}\left(\bar{X}, Q_{l}\right) \rightarrow H^{4}\left(\bar{X}, Q_{l}(2)\right) \simeq Q_{l} / Z_{l} .
$$

Montrons maintenant l'inégalité

$$
\operatorname{dim} H^{4}\left(X, Q_{l} / Z_{l}(3)\right) \geq \operatorname{dim}_{Q_{l}} H^{2}\left(\bar{X}, Q_{l}(2)\right)_{G} .
$$

Or, nous avons l'égalité $\operatorname{dim}_{Q_{l}} H^{2}\left(k, H^{2}\left(\bar{X}, Q_{l}(n)\right)\right)=\operatorname{dim}_{Q_{l}} H^{2}\left(\bar{X}, Q_{l}(n-\right.$ $1))_{G}$ résultant de la dualité de Tate sur le corps $p$-adique $k$; se reportant à la suite exacte (2), il suffit alors de montrer que $H^{3}\left(\bar{X}, Q_{l} / Z_{l}(3)\right)^{G}$ est fini ou, ce qui revient au même, que $H^{3}\left(\bar{X}, Q_{l}(3)\right)^{G}=0$. Or

$$
H^{1}\left(\bar{X}, Q_{l}(2)\right) \stackrel{\text { L.H. }}{\rightarrow} H^{3}\left(\bar{X}, Q_{l}(3)\right)
$$

par Lefschetz dur et $H^{1}\left(\bar{X}, Q_{l}(2)\right)^{G}=0$ par le théorème $5($ a) de [3].

Utilisant (3), on montrerait l'analogue de la proposition 1 pour $n=2$.

Dans le cas particulier où $X$ est simplement connexe, les suites exactes (2) et (3) se simplifient et on obtient:

Proposition 2. Soit $X$ une surface projective, lisse, simplement connexe (par exemple une surface $K-3$ ), définie sur un corps p-adique $k=k_{v}$. Alors

(i) $\operatorname{dim} H^{4}\left(X, Q_{l} / Z_{l}(3)\right)=\operatorname{dim}_{Q_{l}} H^{2}\left(\bar{X}, Q_{l}\right)^{G}$

$$
\leq-\operatorname{ord}_{s=0} L_{v}\left(H^{2}\left(\bar{X}, Q_{l}\right), s\right)
$$

(avec égalité si le Frobenius géométrique $\mathrm{Fr}_{v}$ agit semi-simplement sur $H^{2}\left(\bar{X}, Q_{l}\right)^{I_{v}}$ ou si on admet la conjecture de Serre-Grothendieck).

(ii) $\operatorname{dim} H^{4}\left(X, Q_{l} / Z_{l}(2)\right)_{0}=\operatorname{dim}_{Q_{l}} H^{2}\left(\bar{X}, Q_{l}(1)\right)^{G}$

$$
\leq-\operatorname{ord}_{s=1} L_{v}\left(H^{2}\left(\bar{X}, Q_{l}\right), s\right)
$$

(avec égalité si ...). 
III. Le théorème principal. Soit $X$ une surface projective, lisse, définie sur un corps $p$-adique $k=k_{v}$. Supposons que $X$ admette une réduction semi-stable modulo $p$, i.e. qu'il existe un modèle régulier $\mathcal{X}$ sur spec $\mathcal{O}_{k}$ tel que la fibre spéciale $\mathcal{X}_{s}$ de $\mathcal{X}$ soit un diviseur à croisements normaux : ceci signifie que $\mathcal{X}_{s}$ est réduite, $\mathcal{X}_{s}=\sum Y_{i}$, les $Y_{i}$ étant définies sur $k_{0}$, géométriquement irréductibles, lisses, et se rencontrant transversalement de telle sorte que localement le morphisme structural $\pi: \mathcal{X} \rightarrow \operatorname{spec} \mathcal{O}_{k}$ est définie par $t=x_{1} x_{2} \ldots x_{k}, t$ uniformisante de $\mathcal{O}_{k}$, les $x_{i}$ faisant partie d'un système local de paramètres réguliers de $\mathcal{X}$. Par Deligne [1], $\mathrm{n}^{\mathrm{o}}$ 1.7.2, il existe toujours sur $H^{2}\left(\bar{X}, Q_{l}\right)^{P}, P=\operatorname{ker}\left(I \rightarrow I_{1}\right)$ où $I=I_{v}, I_{1}=1$ - quotient modéré de $I$, une filtration de monodromie locale $W^{M}$. D'autre part, sous les hypothèses précédentes sur $X$, par Rapoport-Zink [4], p. 41 (en particulier, leur proposition 2.13), les valeurs propres associées aux relèvements de l'automorphisme de Frobenius sont pures, ce qui permet de définir une deuxième filtration $W^{\mathrm{Fr}}$ sur $H^{2}\left(\bar{X}, Q_{l}\right)^{P}$, les deux filtrations $W^{M}$ et $W^{\mathrm{Fr}}$ étant alors égales (cf. aussi la proposition 1.7.5 de [1]).

Dans la suite, nous posons

$$
\mathcal{X}_{\bar{s}}^{[p]}=\coprod_{i_{0}<\ldots<i_{p}} \bar{Y}_{i_{0}} \cap \ldots \cap \bar{Y}_{i_{p}}
$$

où $\left(\bar{Y}_{i}\right)_{i \in I}$ désigne la collection des composantes irréductibles de la fibre géométrique spéciale $\mathcal{X}_{\bar{s}}$ de $\mathcal{X}$.

THÉORÈme 1. X satisfaisant aux conditions précédentes, supposons, en outre, les points de $\mathcal{X}_{\bar{s}}^{[2]}$ (= ensemble des points triples de la fibre géométrique spéciale $\left.\mathcal{X}_{\bar{s}}\right)$ rationnels sur le corps résiduel $k_{0}$ de $k$. Alors l'inclusion $H^{2}\left(\bar{X}, Q_{l}\right)^{G} \subseteq W_{0}\left(H^{2}\left(\bar{X}, Q_{l}\right)\right)$ où $W_{0}\left(H^{2}\left(\bar{X}, Q_{l}\right)\right)$ désigne l'ensemble des éléments de poids 0 de $H^{2}\left(\bar{X}, Q_{l}\right)^{P}$ pour $W^{\mathrm{Fr}}$ (ou $W^{M}$, ce qui revient au même) est une égalité et $\operatorname{dim}_{Q_{l}} H^{2}\left(\bar{X}, Q_{l}\right)^{G}=\operatorname{dim}_{Q_{l}} H^{2}\left(|\Gamma|, Q_{l}\right):=h_{2}(|\Gamma|)$ où $|\Gamma|$ désigne le graphe dual de la fibre géométrique spéciale $\mathcal{X}_{\bar{s}}(=$ polyèdre associé aux composantes irréductibles de $\mathcal{X}_{\bar{s}}$ ).

Dém onstration. Il suffit de remarquer que $W_{0}\left(H^{2}\left(\bar{X}, Q_{l}\right)\right)$ se calcule comme l'homologie en degré 2 du complexe (cf. la ligne -4, p. 41 de [4])

$$
H_{0}^{0}\left(\mathcal{X}_{\bar{s}}^{[0]}, Q_{l}\right) \rightarrow H_{1}^{0}\left(\mathcal{X}_{\bar{s}}^{[1]}, Q_{l}\right) \rightarrow H_{2}^{0}\left(\mathcal{X}_{\bar{s}}^{[2]}, Q_{l}\right) \rightarrow 0 .
$$

Ce dernier complexe est aussi le complexe de Čech du graphe dual $|\Gamma|$ de $\mathcal{X}_{\bar{s}}$ ([2], ligne -5 , p. 280). Etant donné l'hypothèse de rationalité faite sur les points de $\mathcal{X}_{\bar{s}}^{[2]}, W_{0}\left(H^{2}\left(\bar{X}, Q_{l}\right)\right)$ est invariante par $G$, d'où l'inclusion $W_{0}\left(H^{2}\left(\bar{X}, Q_{l}\right)\right) \subseteq H^{2}\left(\bar{X}, Q_{l}\right)^{G}$.

Corollaire 1. Sous les hypothèses du théorème $1, L_{v}\left(H^{2}\left(\bar{X}, Q_{l}\right), s\right)$ admet un pôle d'ordre $h_{2}(|\Gamma|)$ en $s=0$. 
En effet, il suffit de remarquer que la valeur propre 1 de $\mathrm{Fr}_{v}$ est semisimple sur $H^{2}\left(\bar{X}, Q_{l}\right)^{I_{v}}$. Or, le sous-espace propre correspondant à une telle valeur propre est contenu dans $W_{0}\left(H^{2}\left(\bar{X}, Q_{l}\right)\right)$ et $\operatorname{Fr}_{v}$ est l'identité sur ce sous-espace puisque, par le théorème $1, W_{0}\left(H^{2}\left(\bar{X}, Q_{l}\right)\right)=H^{2}\left(\bar{X}, Q_{l}\right)^{G}$. On déduit alors de la proposition 1 les égalités suivantes :

$$
-\operatorname{ord}_{s=0} L_{v}\left(H^{2}\left(\bar{X}, Q_{l}\right), s\right)=\operatorname{dim}_{Q_{l}} H^{2}\left(\bar{X}, Q_{l}\right)^{G}=h_{2}(|\Gamma|) .
$$

Remarque 1 . Si les points de $\mathcal{X}_{\bar{s}}^{[2]}$ ne sont pas rationnels sur $k_{0}$, on remplacera $k_{0}$ par une extension finie de $k_{0}$ sur laquelle ils le deviennent : une puissance finie de $\mathrm{Fr}_{v}$ agit trivialement sur $W_{0}\left(H^{2}\left(\bar{X}, Q_{l}\right)\right)$; d'où $\operatorname{Fr}_{v}$ agit semi-simplement sur cet espace et, par la proposition 1 , nous avons encore l'égalité

$$
-\operatorname{ord}_{s=0} L_{v}\left(H^{2}\left(\bar{X}, Q_{l}\right), s\right)=\operatorname{dim}_{Q_{l}} H^{2}\left(\bar{X}, Q_{l}\right)^{G} .
$$

Si les points de $\mathcal{X}_{\bar{s}}^{[2]}$ ne sont pas rationnels sur $k_{0}$, on a la formule

$$
\operatorname{dim}_{Q_{l}} H^{2}\left(\bar{X}, Q_{l}\right)^{G}=\operatorname{dim}_{Q_{l}} H^{2}\left(|\Gamma|, Q_{l}\right)^{G},
$$

$G$ agissant sur $H^{2}\left(|\Gamma|, Q_{l}\right)$ via un quotient fini. On en déduit dans tous les cas l'égalité

$$
-\operatorname{ord}_{s=0} L_{v}\left(H^{2}\left(\bar{X}, Q_{l}\right), s\right)=\operatorname{dim}_{Q_{l}} H^{2}\left(|\Gamma|, Q_{l}\right)^{G},
$$

$G$ agissant via un quotient fini.

Corollaire 2. Soit $X$ une surface $K$-3 définie sur un corps p-adique $k=k_{v}$. Supposons que $X$ admette un modèle régulier $\mathcal{X}$ sur $\operatorname{spec} \mathcal{O}_{k}$. Alors

(a) si $\mathcal{X}$ admet une dégénérescence standard de type $A$ ou $B$ au sens de Shafarevich-Kulikov [5], $L_{v}\left(H^{2}\left(\bar{X}, Q_{l}\right), s\right)$ n'a pas de pôle en $s=0$,

(b) si $\mathcal{X}$ admet une dégénérescence standard de type $C$ au sens de loc. cit., les points de $\mathcal{X}_{\bar{s}}^{[2]}$ étant rationnels sur le corps résiduel $k_{0}$ de $k$, $L_{v}\left(H^{2}\left(\bar{X}, Q_{l}\right), s\right)$ admet un pôle en $s=0$ d'ordre égal à 1 .

Comme dans le corollaire 1, la valeur propre 1 de $\mathrm{Fr}_{v}$ est semi-simple sur $H^{2}\left(\bar{X}, Q_{l}\right)^{I_{v}}$. Par la proposition $2(\mathrm{i})$, on a alors

$$
-\operatorname{ord}_{s=0} L_{v}\left(H^{2}\left(\bar{X}, Q_{l}\right), s\right)=\operatorname{dim}_{Q_{l}} H^{2}\left(\bar{X}, Q_{l}\right)^{G}
$$

et, par le théorème 1 ,

$$
\operatorname{dim}_{Q_{l}} H^{2}\left(\bar{X}, Q_{l}\right)^{G}=h_{2}(|\Gamma|)= \begin{cases}0 & \text { dans le cas (a) } \\ 1 & \text { dans le cas (b) }\end{cases}
$$

(par définition des dégénérescences standard [5], p. 719, on sait que $|\Gamma|$ a le type d'homotopie de $S^{2}$ dans le cas (b) et que $h_{2}(|\Gamma|)=0$ dans le cas (a)).

Remarque 2. En utilisant la proposition 2(ii), on pourrait aussi montrer que $L_{v}\left(H^{2}\left(\bar{X}, Q_{l}\right), s\right)$ n'admet pas de pôle en $s=1, X$ étant une 
surface K-3 comme dans le corollaire 2 et cela quel que soit son type de dégénérescence.

Corollaire 3. Soit $X$ une surface abélienne définie sur un corps padique $k$. Faisons les hypothèses suivantes sur $X$ :

(i) $X$ est de type III, i.e. la fibre spéciale géométrique du modèle de Néron de $X$ est un tore $T$ isomorphe au produit $G_{m} \times G_{m}$.

(ii) $T$ est déployé sur le corps résiduel $k_{0}$ de $k$. Alors $L_{v}\left(H^{2}\left(\bar{X}, Q_{l}\right), s\right)$ admet un pôle d'ordre 1 en $s=0$.

Démonstration. On procède comme dans les corollaires 1 et 2 en remarquant que, sous les hypothèses du corollaire $3, h_{2}(|\Gamma|)=1$.

R e mar qu e 3. Le corollaire 3 précédent est à rapprocher du théorème $5(\mathrm{~b}), \mathrm{n}^{\mathrm{o}} 7$ de [3].

IV. Un résultat global. Nous supposerons dans ce paragraphe que $k$ est un corps de nombres. Soit $X$ une surface K-3 définie sur $k$ satisfaisant aux conditions suivantes :

(i) $X$ admet un modèle régulier $\mathcal{X} \operatorname{sur} \operatorname{spec} \mathcal{O}_{k}$,

(ii) toutes les dégénérescences de $\mathcal{X}$ en les places de mauvaise réduction sont standard de type A, B, C au sens de Shafarevich-Kulikov [5] comme dans le corollaire 2 au théorème 1 .

Par un théorème de $\mathrm{O}$. Gabber, on sait que, pour presque tout $l$, $H^{2}\left(\bar{X}, Z_{l}\right)$ est sans torsion; on en déduit que, pour presque tout $l$, $H^{2}\left(\bar{X}, Q_{l} / Z_{l}(3)\right)$ est aussi sans torsion, donc que $H^{2}\left(\bar{X}, Q_{l} / Z_{l}(3)\right)$ s'identifie, pour presque tout $l$, à sa partie divisible

$$
\widetilde{H}^{2}\left(\bar{X}, Q_{l} / Z_{l}(3)\right)=l-\operatorname{div} H^{2}\left(\bar{X}, Q_{l} / Z_{l}(3)\right) .
$$

D'où :

$$
\begin{aligned}
H^{4}\left(X, Q_{l} / Z_{l}(3)\right)_{0} & \simeq H^{2}\left(k, H^{2}\left(\bar{X}, Q_{l} / Z_{l}(3)\right)\right) \\
& \simeq H^{2}\left(k, \widetilde{H}^{2}\left(\bar{X}, Q_{l} / Z_{l}(3)\right)\right) \text { pour presque tout } l .
\end{aligned}
$$

Or, par le théorème 3(d) de [3], $S$ désignant l'ensemble des places non archimédiennes de $k$ où $\mathcal{X}$ admet mauvaise réduction, $l$ étant supposé $\neq 2$, on a

$$
H^{2}\left(k, \widetilde{H}^{2}\left(\bar{X}, Q_{l} / Z_{l}(3)\right)\right) \simeq \bigoplus_{v \in S} H^{2}\left(k_{v}, \widetilde{H}^{2}\left(\bar{X}, Q_{l} / Z_{l}(3)\right)\right)
$$

D'où

$$
H^{4}\left(X, Q_{l} / Z_{l}(3)\right)_{0} \simeq \bigoplus_{v \in S} H^{2}\left(k_{v}, \widetilde{H}^{2}\left(\bar{X}, Q_{l} / Z_{l}(3)\right)\right) \quad \text { pour presque tout } l \text {. }
$$


Par les calculs faits précédemment au §I et dans la démonstration du corollaire 2 au théorème 1 , pour $v \in S$, compte-tenu de (ii),

$$
H^{2}\left(k_{v}, \widetilde{H}^{2}\left(\bar{X}, Q_{l} / Z_{l}(3)\right)\right) \begin{cases}\simeq Q_{l} / Z_{l} & \text { si } v \text { est de type C, } \\ =0 & \text { si } v \text { est de type B. }\end{cases}
$$

On peut donc énoncer le

ThÉORÈme 2. Soit $X$ une surface K-3 définie sur un corps de nombres et satisfaisant aux conditions (i) et (ii) précédentes. Alors

$\operatorname{dim} H^{4}\left(X, Q_{l} / Z_{l}(3)\right)=\#\{v \in S$ où la réduction est de type $C\}$.

\section{Références}

[1] P. Deligne, La conjecture de Weil. II, Inst. Hautes Études Sci. Publ. Math. 52 (1980), 137-252.

[2] J. C. Douai, Dégénérescence des surfaces et principe de Hasse, J. Algebra 152 (1992), 269-288.

[3] U. Jannsen, On the l-adic cohomology of varieties over number fields and its Galois cohomology, dans : Galois Group over $\mathbb{Q}$, Y. Ihara, K. A. Ribet and J. P. Serre (eds.), Springer, Berlin, 1989, 315-360.

[4] M. Rapoport und T. Zink, Über die lokale Zetafunktion von Shimuravarietäten. Monodromiefiltration und verschwindende Zyklen in ungleicher Charakteristik, Invent. Math. 68 (1982), 21-101.

[5] I. R. Shafarevich, The influence of height on degenerations of algebraic surfaces of type K-3 (with A. V. Rudakov and T. Zink), Collected Math. Papers, Springer, 1989, 715-731.

UFR MATHÉMATIQUES - M

UNIVERSITÉ DES SCIENCES ET TECHNIQUES DE LILLE

F-59655 VILLENEUVE D'ASCQ CEDEX, FRANCE 